
\title{
28 Research Square \\ The Efficacy of Low-dose Aspirin in Pregnancy Among Women in Malaria-endemic Countries
}

Melissa Bauserman ( $\square$ Melissa_bauserman@med.unc.edu )

University of North Carolina at Chapel Hill

\section{Sequoia Leuba}

University of North Carolina at Chapel Hill

Jennifer Hemingway-Foday

RTI International

\section{Tracy L. Nolen}

RTI International

Janet Moore

RTI International

Elizabeth M. McClure

RTI International

Adrien Lokangaka

Kinshasa School of Public Health

Antoinette Tsehfu

Kinshasa School of Public Health

Jackie Patterson

University of North Carolina at Chapel Hill

Edward Liechty

Indiana University School of Medicine, University of Indiana

Fabian Esamai

Moi University School of Medicine

Wally Carlo

University of Alabama at Birmingham

\section{Elwyn Chomba}

University Teaching Hospital

\section{Robert L. Goldenberg}

Columbia University School of Medicine

\section{Sarah Saleem}

Aga Khan University

\section{Saleem Jesani}

Aga Khan University

Marion Koso-Thomas 
Eunice Kennedy Shriver National Institute of Child Health and Human Development

\section{Matthew Hoffman}

Christiana Care

\section{Richard Derman}

Thomas Jefferson University

\section{Steve Meshnick}

University of North Carolina at Chapel Hill

\section{Carl Bose}

University of North Carolina at Chapel Hill

\section{Research Article}

Keywords: malaria, pregnancy, premature birth, perinatal mortality

Posted Date: September 3rd, 2021

DOl: https://doi.org/10.21203/rs.3.rs-803905/v1

License: (c) (i) This work is licensed under a Creative Commons Attribution 4.0 International License. Read Full License

Version of Record: A version of this preprint was published at BMC Pregnancy and Childbirth on April 10th, 2022. See the published version at https://doi.org/10.1186/s12884-022-04652-9. 


\section{Abstract}

\section{Background}

Low dose aspirin (LDA) is an effective strategy to reduce preterm birth. However, LDA might have differential effects globally, based on the etiology of preterm birth. In some regions, malaria in pregnancy could be an important modifier of LDA on birth outcomes and anemia.

\section{Methods}

This is a sub-study of the ASPIRIN trial, a multi-national, randomized, placebo controlled trial evaluating LDA effect on preterm birth. We enrolled a convenience sample of women in the ASPIRIN trial from the Democratic Republic of Congo (DRC), Kenya and Zambia. We used quantitative polymerase chain reaction to detect malaria. We calculated crude prevalence proportion ratios (PRs) for LDA by malaria for outcomes, and regression modelling to evaluate effect measure modification. We evaluated hemoglobin in late pregnancy based on malaria infection in early pregnancy.

\section{Results}

1,446 women were analyzed, with a malaria prevalence of $63 \%$ in the DRC site, $38 \%$ in the Kenya site, and $6 \%$ in the Zambia site. Preterm birth occurred in 83 (LDA) and 90 (placebo) women, (PR 0.92, 95\% Cl 0.70, 1.22), without interaction between LDA and malaria $(p=0.75)$. Perinatal mortality occurred in 41 (LDA) and 43 (placebo) pregnancies, (PR 0.95, 95\% $\mathrm{Cl} 0.63,1.44)$, with an interaction between malaria and LDA $(p=0.014)$. Hemoglobin was similar by malaria and LDA status.

\section{Conclusions}

Malaria in early pregnancy did not modify the effects of LDA on preterm birth, but modified the effect of LDA on perinatal mortality. This effect measure modification deserves continued study as LDA is used in malaria endemic regions.

\section{Background}

Preterm birth is a major cause of neonatal mortality, and infants born in low- and middle-income countries (LMICs) are particularly vulnerable. Recent trials demonstrate that low dose aspirin (LDA) is an effective strategy to reduce preterm birth.(1-3) However, LDA might have differential effects in global regions, based on the etiology of preterm birth. The Eunice Kennedy Shriver National Institute for Child Health and Human Development (NICHD) Global Network for Women's and Children's Health Research (GN) recently completed the ASPIRIN trial, a multi-country trial of the effects of LDA on preterm birth. $(3,4)$ While, this study was not powered to investigate efficacy in individual sites, site-specific data did not demonstrate a reduction in the risk of preterm birth for LDA in the Democratic Republic of the Congo (DRC).(3) One feature that distinguished this site from others in the ASPIRIN trial was high malaria prevalence. 
Malaria infections during pregnancy are an important cause of preterm birth in many LMICs, especially sub-Saharan African countries where malaria infection in pregnancy is common.(5) Malaria is associated with up to $36 \%$ of all preterm or low birth weight (LBW) infants born in malaria endemic areas.(6) Malaria treatment or prophylaxis in the latter half of pregnancy improves outcomes.(7) Studies also suggest that malaria may exert deleterious effects early in pregnancy that lead to preterm birth, stillbirth, pregnancy loss, anemia or other adverse pregnancy outcomes.(7-9) Malaria infections during early pregnancy are associated with abnormal development of the placental circulation that may result in hypertensive disorders, intrauterine growth restriction (IUGR) and prematurity.(5) Early malaria infections may cause both local and systemic inflammation leading to poor placentation that leads to these adverse pregnancy outcomes.

LDA is thought to reduce inflammation and thrombosis that leads to placental dysfunction, preterm birth, preeclampsia and IUGR.(10,11) Malaria could be an important effect modifier of the relationship between LDA and preterm birth in malaria-endemic regions. For example, malaria may reduce the beneficial effects of LDA by increasing inflammation. Conversely, LDA may reduce the adverse impact of malaria in early pregnancy by reducing placental inflammation caused by malaria.

LDA treatment to prevent preterm birth has important public health implications, and might be adopted in LMICs, including those countries in malaria-endemic regions. Therefore, there is an urgent need to understand the relationships between LDA and malaria in early pregnancy on maternal and neonatal health. The objectives of this paper were to investigate if LDA has a differential effect in areas where malaria is prevalent and determine whether malaria infection interacts with the effects of LDA on pregnancy outcomes. Furthermore, we also investigated the relationships between malaria, LDA and anemia in pregnancy to determine if a potential effect of malaria was mediated through anemia, a common morbidity of the disease.

\section{Methods}

This study was a sub-study of the ASPIRIN (Aspirin Supplementation for Pregnancy Indicated Risk Reduction In Nulliparas) trial.(4) The ASPIRIN trial was a multi-national, randomized, double-masked, placebo controlled trial to evaluate the effects of LDA ( $81 \mathrm{mg}$ daily) in nulliparous women on the outcome of preterm birth (birth before 37 weeks gestational age). Across 8 research sites in LMICs, women were randomized in a 1:1 ratio to receive LDA (treatment) or an identically-appearing placebo (control).

Pregnant women were eligible for the ASPIRIN trial if they were nulliparous, between 14-40 years of age, had pregnancies characterized by a gestational age between 6 weeks and 0 days and 13 weeks and 6 days (confirmed by ultrasound), blood pressure $<140 / 90 \mathrm{mmHg}$, hemoglobin $>7.0 \mathrm{~g} / \mathrm{dL}$, a live singleton fetus, and absence of fetal anomaly. Women were excluded who had allergy or contraindication to aspirin, previous aspirin use for more than 7 days, a history of $\geq 2$ first-trimester losses, or the presence of diabetes or hypertension.(4) 
For the sub-study, we enrolled a convenience sample of women participating in the ASPIRIN trial from 4 of the $8 \mathrm{GN}$ sites. The four sites were chosen based on the potential for malaria affecting the pregnancies in the study regions, and represented a range of malaria endemicity (high and low). These 4 sites were located in the DRC (North and South Ubangi Provinces), Kenya (Western region), Pakistan (near the city of Karachi), and Zambia (south and east of the capital city of Lusaka).

Women enrolled in the malaria sub-study had blood collected at times coincident with blood collection in the ASPIRIN trial. Blood was collected at enrollment (6-13 6/7 weeks gestation) and during the third trimester (between 26-30 weeks gestation), using dried blood spot techniques. All blood spot cards were shipped to a central laboratory, the Meshnick Laboratory at the University of North Carolina (U.S.) for analyses. The PCR results were performed in batches, in most cases, after the conclusion of pregnancies, therefore the PCR research results were not available to guide treatment decisions during pregnancy. Rapid diagnostic tests (RDTs) were also performed for women enrolled at the Pakistan, Zambia and Kenya sites, and women were treated according to local standards based on the results. No RDTs were performed at the DRC site. Each sample was tested in duplicate for $P$. falciparum lactate dehydrogenase (pfldh) by quantitative polymerase chain reaction (qPCR). Because malaria parasites were not detected in the samples from Pakistan, the women enrolled from the Pakistani site were excluded from subsequent analyses.

We defined primary and secondary efficacy outcomes as specified in the ASPIRIN trial. Therefore, preterm delivery was defined as delivery after 20 weeks, but before $370 / 7$ weeks gestation. We defined hypertensive disorders to include pre-eclampsia, eclampsia, and gestational hypertension. We defined small for gestational age as a birth weight less than $10 \%$ of the INTERGROWTH-21st growth standard. (12) We defined perinatal mortality as stillbirths and deaths in the perinatal period from 20 weeks gestation to 7 days post-partum. Additionally, we used a priori specifications for other maternal outcomes of interest, such as maternal vaginal bleeding, antepartum/postpartum hemorrhage, maternal mortality and a combined category of preterm and hypertensive disorders. We used a priori defined specifications for other fetal outcomes of interest, including preterm delivery < 34 weeks, birthweight $<2500$ grams, birthweight $<1500$ grams and stillbirth. We also defined malaria in late pregnancy as an infection in women for whom we collected a secondary blood sample between 26-30 weeks that was positive for malaria by qPCR.

We performed descriptive statistics for bivariate comparisons. Crude prevalence proportion ratios (PRs) were calculated for LDA treatment by $P$. falciparum infection in the first trimester by each variable using stratified tables for all sites and for each site individually. Poisson models were developed to evaluate effect measure modification of malaria on the effect of LDA in regards to maternal and fetal outcomes of interest with terms included for treatment, malaria in the first trimester, site, and the interaction term of malaria in the first trimester and LDA treatment. Similar models were constructed separately for each site to estimate the prevalence ratio of preterm birth and perinatal mortality with terms included for treatment, malaria in the first trimester, and the interaction term of malaria in the first trimester and LDA treatment. 
We used prevalence ratios (PRs) to investigate the relationship between early pregnancy malaria status and late pregnancy hemoglobin levels. Hemoglobin levels were measured by point of care testing devices in the third trimester (between 26-30 weeks). We used multiple cutoffs for hemoglobin levels ( $\leq 8 \mathrm{~g} / \mathrm{dL}$, $\leq 9 \mathrm{~g} / \mathrm{dL}, \leq 10 \mathrm{~g} / \mathrm{dL}$, and $\leq 11 \mathrm{~g} / \mathrm{dL}$ ) to assess whether malaria in the first trimester was an effect measure modifier for the relationship between LDA treatment and hemoglobin levels in late pregnancy. Comparisons were limited to observations without missing data for each variable. All analyses were performed using the R statistical platform (version 4.0.2) and SAS version 9.4 (SAS Institute, Cary, NC, USA).

This study was approved by the relevant ethics committees at the institutions conducting the study at each site prior to the initiation of study activities. The study was also approved by the ethics committees at the partner U.S.-based institutions (University of North Carolina at Chapel Hill, Columbia University, University of Alabama at Birmingham and Indiana University) and by RTI International, the data coordinating center. All women provided informed consent prior to their participation in the sub-study. The ASPIRIN trial was registered in clinicaltrials.gov (NCT02409680).

\section{Results}

We included 1,446 pregnant women in our analyses; 469 (32\%) from DRC, 642 (44\%) from Kenya, and 335 (23\%) from Zambia (Fig. 1).

The women in the DRC site had the highest prevalence of malaria in early pregnancy $(297 / 469,63 \%)$, followed by the women in the Kenya site $(244 / 642,38 \%)$, then the Zambia site $(21 / 335,6 \%)$. Half of the women $(n=724,50 \%)$ were randomized to the LDA (treatment) arm. Of the women who received LDA, $39 \%(279 / 724)$ had malaria in early pregnancy, equal to $39 \%$ of women $(283 / 722)$ with malaria in early pregnancy who were randomized to placebo (Table 1). 
Table 1

Baseline Characteristics of Study Population by Treatment Arm and Malaria Status in Early Pregnancy

\section{ALL COUNTRIES}

\begin{tabular}{|c|c|c|c|c|}
\hline \multirow{2}{*}{ Variable } & \multicolumn{2}{|c|}{$\operatorname{LDA}(\mathrm{N}=724)$} & \multicolumn{2}{|c|}{ PLACEBO (N = 722) } \\
\hline & $\begin{array}{l}\text { Malaria } \\
\text { negative }\end{array}$ & $\begin{array}{l}\text { Malaria } \\
\text { positive }\end{array}$ & $\begin{array}{l}\text { Malaria } \\
\text { negative }\end{array}$ & $\begin{array}{l}\text { Malaria } \\
\text { positive }\end{array}$ \\
\hline Randomized, N (\%) & $445(61)$ & 279 (39) & $439(61)$ & $283(39)$ \\
\hline \multicolumn{5}{|c|}{ Maternal age (years), N (\%) } \\
\hline$<20$ & $225(50 \cdot 6)$ & $209(74 \cdot 9)$ & $250(56 \cdot 9)$ & $206(72 \cdot 8)$ \\
\hline $20-29$ & $214(48 \cdot 1)$ & $69(24 \cdot 7)$ & $185(42 \cdot 1)$ & $74(26 \cdot 1)$ \\
\hline$>29$ & $6(1 \cdot 3)$ & $1(0 \cdot 4)$ & $4(0 \cdot 9)$ & $3(1 \cdot 1)$ \\
\hline Median (P25, P75) & $\begin{array}{l}19 \cdot 0(18 \cdot 0 \\
22 \cdot 0)\end{array}$ & $\begin{array}{l}18 \cdot 0(17 \cdot 0 \\
19 \cdot 5)\end{array}$ & $\begin{array}{l}19 \cdot 0(18 \cdot 0 \\
21 \cdot 0)\end{array}$ & $\begin{array}{l}18 \cdot 0(17 \cdot 0, \\
20 \cdot 0)\end{array}$ \\
\hline \multicolumn{5}{|c|}{$\begin{array}{l}\text { Projected gestational age at } \\
\text { enrollment (weeks, days), N (\%) }\end{array}$} \\
\hline $6,0-7,6$ & $63(14 \cdot 2)$ & $30(10 \cdot 8)$ & $72(16 \cdot 4)$ & $26(9 \cdot 2)$ \\
\hline $8,0-9,6$ & $134(30 \cdot 1)$ & $75(26 \cdot 9)$ & $114(26 \cdot 0)$ & $76(26 \cdot 9)$ \\
\hline $10,0-10,6$ & $66(14 \cdot 8)$ & $33(11 \cdot 8)$ & $57(13 \cdot 0)$ & $31(11 \cdot 0)$ \\
\hline $11,0-11,6$ & $47(10 \cdot 6)$ & $48(17 \cdot 2)$ & $66(15 \cdot 0)$ & $54(19 \cdot 1)$ \\
\hline $12,0-13,6$ & $135(30 \cdot 3)$ & $93(33 \cdot 3)$ & $130(29 \cdot 6)$ & 96 (33.9) \\
\hline Median (P25, P75) & $\begin{array}{l}10 \cdot 3(8 \cdot 7 \\
12 \cdot 3)\end{array}$ & $\begin{array}{l}11 \cdot 0(9 \cdot 1 \\
12 \cdot 4)\end{array}$ & $\begin{array}{l}10 \cdot 6(8 \cdot 4 \\
12 \cdot 1)\end{array}$ & $\begin{array}{l}11 \cdot 1(9 \cdot 0 \\
12 \cdot 4)\end{array}$ \\
\hline \multicolumn{5}{|c|}{ Maternal education, $\mathrm{N}(\%)$} \\
\hline No formal & $20(4 \cdot 5)$ & $25(9 \cdot 0)$ & $19(4 \cdot 3)$ & $24(8 \cdot 5)$ \\
\hline Primary & $58(13 \cdot 0)$ & $84(30 \cdot 1)$ & $60(13 \cdot 7)$ & $97(34 \cdot 3)$ \\
\hline Secondary & $334(75 \cdot 1)$ & $158(56 \cdot 6)$ & $327(74 \cdot 5)$ & $156(55 \cdot 1)$ \\
\hline University + & $33(7 \cdot 4)$ & $12(4 \cdot 3)$ & $33(7 \cdot 5)$ & $6(2 \cdot 1)$ \\
\hline \multicolumn{5}{|c|}{ Maternal height (cm), N } \\
\hline Mean (StdDev) & $157 \cdot 0(7 \cdot 5)$ & $155 \cdot 6(8 \cdot 0)$ & $157 \cdot 0(7 \cdot 4)$ & $155 \cdot 2(7 \cdot 9)$ \\
\hline Median (P25, P75) & $\begin{array}{l}158 \cdot 0 \\
(152 \cdot 5 \\
162 \cdot 0)\end{array}$ & $\begin{array}{l}156 \cdot 0 \\
(150 \cdot 2 \\
161 \cdot 0)\end{array}$ & $\begin{array}{l}157 \cdot 0 \\
(152 \cdot 5 \\
161 \cdot 9)\end{array}$ & $\begin{array}{l}155 \cdot 0 \\
(150 \cdot 0, \\
160 \cdot 0)\end{array}$ \\
\hline
\end{tabular}




\section{ALL COUNTRIES}

\section{Maternal weight $(\mathrm{kg}), \mathrm{N}$}

Mean (StdDev)

Median (P25, P75)

Maternal BMI (kg/m2), N

Mean (StdDev)

Median (P25, P75)

\section{Antenatal care visits, $\mathbf{N}$}

Mean (StdDev)

Median (P25, P75)

\section{Delivery attendant, $\mathbf{N}(\%)$}

Physician

Nurse/nurse midwife

Traditional birth attendant

Family/Self/Other

\section{Delivery location, $\mathbf{N}(\%)$}

Hospital

Clinic/health center

Home/Other

\section{Delivery mode, $\mathbf{N}(\%)$}

Vaginal

C-section

\section{Site, N (\%)}

DRC

Kenya

Zambia
$55 \cdot 0(8 \cdot 1)$

$54 \cdot 0(50 \cdot 0$, $59 \cdot 0)$

$22 \cdot 4(3 \cdot 4)$

$21 \cdot 1(20 \cdot 0$, 24.0)
$53 \cdot 0(7 \cdot 4)$

$52 \cdot 2(48 \cdot 0$, $58 \cdot 0)$
$55 \cdot 0(8 \cdot 3)$

$54 \cdot 0(50 \cdot 0$, $60 \cdot 0)$
$52 \cdot 3(7 \cdot 5)$

$52 \cdot 0(47 \cdot 0$, $56 \cdot 8)$
$21 \cdot 8(3 \cdot 1)$

$21.5(19 \cdot 5$, $21 \cdot 7(19 \cdot 7$, 23.4)

$21 \cdot 9(20 \cdot 0$, 24.1) 23.3)
$4 \cdot 2(1 \cdot 1)$

$4 \cdot 0(4 \cdot 0$,

$5 \cdot 0)$ $5 \cdot 0)$

4.0 (4.0,

$5 \cdot 0)$

$24(5 \cdot 4)$

$391(87 \cdot 9)$

$19(4 \cdot 3)$

$11(2 \cdot 5)$

$113(25 \cdot 4) \quad 58(20 \cdot 8)$

$296(66 \cdot 5)$

$36(8 \cdot 1)$

$426(95 \cdot 7)$

$19(4 \cdot 3)$
$9(3 \cdot 2)$

$241(86 \cdot 4)$

$22(7 \cdot 9)$

$7(2 \cdot 5)$

$180(64 \cdot 5)$

$41(14 \cdot 7)$
$18(4 \cdot 1)$

$383(87 \cdot 2)$

$23(5 \cdot 2)$

$15(3 \cdot 4)$

$122(27 \cdot 8)$ $270(61 \cdot 5)$

$47(10 \cdot 7)$

$422(96 \cdot 1)$

17 (3.9)
$3.9(1 \cdot 2)$

4.0 (3.0, $5 \cdot 0)$ 
Baseline characteristics were similar among women who received LDA vs placebo, and among women who had malaria in early pregnancy vs those who did not, in terms of gestational age at enrollment, maternal height, weight, body mass index (BMI), antenatal care visits, delivery attendant, delivery location and mode of delivery (Table 1 ). Women with malaria in early pregnancy trended toward younger maternal ages and fewer years of formal education. The majority of all mothers had deliveries attended by nurses/midwives, delivered in clinics or health centers and had vaginal deliveries. The median number of antenatal care (ANC) visits was four.

Preterm birth occurred in 83 women in the LDA group and 90 women in the placebo group (PR 0.92, 95\% Cl 0.70, 1.22) (Table 2). 
Table 2

Primary and secondary efficacy outcomes based on infection with malaria in early pregnancy

\begin{tabular}{|c|c|c|c|c|c|c|c|}
\hline \multirow[t]{2}{*}{ Variable } & \multicolumn{2}{|l|}{ Overall } & \multicolumn{2}{|c|}{ Malaria Negative } & \multicolumn{2}{|c|}{ Malaria Positive } & \multirow{2}{*}{$\begin{array}{l}\text { Aspirin - } \\
\text { Malaria } \\
\text { Interaction }\end{array}$} \\
\hline & $\mathrm{n} / \mathrm{N}(\%)$ & $\begin{array}{l}\text { PR } \\
\text { (95\% } \\
\text { Cl) }\end{array}$ & $\begin{array}{l}\mathrm{n} / \mathrm{N} \\
(\%)\end{array}$ & $\begin{array}{l}\text { PR } \\
\text { (95\% } \\
\text { Cl) }\end{array}$ & $\begin{array}{l}\mathrm{n} / \mathrm{N} \\
(\%)\end{array}$ & $\begin{array}{l}\text { PR } \\
(95 \% \\
\text { Cl) }\end{array}$ & \\
\hline
\end{tabular}

\section{Primary Outcome}

Preterm delivery

\begin{tabular}{|c|c|c|c|c|c|c|c|}
\hline Aspirin & $\begin{array}{l}83 / 724 \\
(11)\end{array}$ & $\begin{array}{l}0.92 \\
(0.70 \\
1.22)\end{array}$ & $\begin{array}{l}38 / 445 \\
(9)\end{array}$ & $\begin{array}{l}0.89 \\
(0.59 \\
1.36)\end{array}$ & $\begin{array}{l}45 / 279 \\
(16)\end{array}$ & $\begin{array}{l}0.95 \\
(0.66, \\
1.38)\end{array}$ & 0.75 \\
\hline Placebo & $\begin{array}{l}90 / 722 \\
(12)\end{array}$ & & $\begin{array}{l}42 / 439 \\
(10)\end{array}$ & & $\begin{array}{l}48 / 283 \\
(17)\end{array}$ & & \\
\hline
\end{tabular}

Hypertensive

disorders

\begin{tabular}{|c|c|c|c|c|c|c|}
\hline Aspirin & $10 / 724(1)$ & $\begin{array}{l}1 \cdot 11 \\
(0 \cdot 45 \\
2 \cdot 71)\end{array}$ & $\begin{array}{l}8 / 445 \\
(2)\end{array}$ & $\begin{array}{l}0.88 \\
(0.34 \\
2 \cdot 25)\end{array}$ & $\begin{array}{l}2 / 279 \\
(1)\end{array}$ & $\begin{array}{l}5 \cdot 07 \\
(0 \cdot 24 \\
105 \cdot 17)\end{array}$ \\
\hline
\end{tabular}

Placebo

9/722(1)

$9 / 439$

(2)

$0 / 283$

(0)

Small for

gestational age

\begin{tabular}{|c|c|c|c|c|c|c|}
\hline Aspirin & $\begin{array}{l}89 / 686 \\
(13)\end{array}$ & $\begin{array}{l}1 \cdot 11 \\
(0.84 \\
1 \cdot 48)\end{array}$ & $\begin{array}{l}58 / 421 \\
(14)\end{array}$ & $\begin{array}{l}1 \cdot 21 \\
(0.84, \\
1 \cdot 73)\end{array}$ & $\begin{array}{l}31 / 265 \\
(12)\end{array}$ & $\begin{array}{l}0.97 \\
(0.61, \\
1.54)^{\prime}\end{array}$ \\
\hline Placebo & $\begin{array}{l}78 / 668 \\
(12)\end{array}$ & & $\begin{array}{l}46 / 403 \\
(11)\end{array}$ & & $\begin{array}{l}32 / 265 \\
(12)\end{array}$ & \\
\hline
\end{tabular}

Perinatal

mortality

\begin{tabular}{|c|c|c|c|c|c|c|}
\hline Aspirin & $41 / 724$ (6) & $\begin{array}{l}0.95 \\
(0.63, \\
1.44)\end{array}$ & $\begin{array}{l}16 / 445 \\
(4)\end{array}$ & $\begin{array}{l}0.56 \\
(0.31 \\
1.03)\end{array}$ & $\begin{array}{l}25 / 279 \\
\text { (9) }\end{array}$ & $\begin{array}{l}1 \cdot 69 \\
(0 \cdot 91, \\
3 \cdot 14)\end{array}$ \\
\hline
\end{tabular}

Placebo

$43 / 722(6)$

$28 / 439$

(6)

$15 / 283$

(5)

\section{Other Maternal Outcomes of Interest}

Vaginal bleeding

Aspirin

$$
\begin{array}{ll}
12 / 713(2) \quad & 0 \cdot 86 \\
& (0 \cdot 40 \\
& 1 \cdot 85)
\end{array}
$$

$11 / 437$

(3)

0.91

$(0 \cdot 41$,

2.05)

$1 / 276$

(0)

$\begin{array}{ll}0.51 & 0.64 \\ (0 \cdot 05, & \\ 5 \cdot 56) & \end{array}$




\begin{tabular}{|c|c|c|c|c|c|c|c|}
\hline \multirow[t]{2}{*}{ Variable } & \multicolumn{2}{|l|}{ Overall } & \multicolumn{2}{|c|}{ Malaria Negative } & \multicolumn{2}{|c|}{ Malaria Positive } & \multirow{2}{*}{$\begin{array}{l}\text { Aspirin - } \\
\text { Malaria } \\
\text { Interaction } \\
\text { p-value }\end{array}$} \\
\hline & $\mathrm{n} / \mathrm{N}(\%)$ & $\begin{array}{l}\text { PR } \\
(95 \% \\
\text { Cl) }\end{array}$ & $\begin{array}{l}\mathrm{n} / \mathrm{N} \\
(\%)\end{array}$ & $\begin{array}{l}\text { PR } \\
(95 \% \\
\mathrm{Cl})\end{array}$ & $\begin{array}{l}n / N \\
(\%)\end{array}$ & $\begin{array}{l}\text { PR } \\
(95 \% \\
\text { Cl) }\end{array}$ & \\
\hline Placebo & $14 / 715(2)$ & & $\begin{array}{l}12 / 435 \\
(3)\end{array}$ & & $\begin{array}{l}2 / 280 \\
(1)\end{array}$ & & \\
\hline \multicolumn{8}{|l|}{$\begin{array}{l}\text { Antepartum } \\
\text { hemorrhage }\end{array}$} \\
\hline Aspirin & $4 / 721(1)$ & $\begin{array}{l}8.95 \\
(0 \cdot 48 \\
165 \cdot 94)\end{array}$ & $\begin{array}{l}4 / 442 \\
(1)\end{array}$ & $\begin{array}{l}8 \cdot 86 \\
(0 \cdot 48 \\
164 \cdot 04)\end{array}$ & $\begin{array}{l}0 / 279 \\
(0)\end{array}$ & $\begin{array}{l}1 \cdot 01 \\
(0 \cdot 02 \\
50 \cdot 76)\end{array}$ & -- \\
\hline Placebo & 0/717 (0) & & $\begin{array}{l}0 / 435 \\
(0)\end{array}$ & & $\begin{array}{l}0 / 282 \\
(0)\end{array}$ & & \\
\hline \multicolumn{8}{|l|}{$\begin{array}{l}\text { Postpartum } \\
\text { hemorrhage }\end{array}$} \\
\hline Aspirin & $6 / 724(1)$ & $\begin{array}{l}2.99 \\
(0 \cdot 61 \\
14 \cdot 77)\end{array}$ & $\begin{array}{l}2 / 445 \\
(0)\end{array}$ & $\begin{array}{l}4 \cdot 93 \\
(0 \cdot 24 \\
102 \cdot 46)\end{array}$ & $\begin{array}{l}4 / 279 \\
(1)\end{array}$ & $\begin{array}{l}2 \cdot 03 \\
(0 \cdot 37 \\
10 \cdot 99)\end{array}$ & -- \\
\hline Placebo & $2 / 722(0)$ & & $\begin{array}{l}0 / 439 \\
(0)\end{array}$ & & $\begin{array}{l}2 / 283 \\
(0)\end{array}$ & & \\
\hline \multicolumn{8}{|c|}{$\begin{array}{l}\text { Maternal } \\
\text { mortality through } \\
42 \text { days }\end{array}$} \\
\hline Aspirin & $0 / 724(0)$ & $\begin{array}{l}0.33 \\
(0 \cdot 02 \\
8 \cdot 15)\end{array}$ & $\begin{array}{l}0 / 445 \\
(0)\end{array}$ & $\begin{array}{l}0.33 \\
(0.02 \\
8 \cdot 05)\end{array}$ & $\begin{array}{l}0 / 279 \\
(0)\end{array}$ & $\begin{array}{l}1 \cdot 01 \\
(0 \cdot 02 \\
50 \cdot 94)\end{array}$ & - \\
\hline Placebo & $1 / 722(0)$ & & $\begin{array}{l}1 / 439 \\
(0)\end{array}$ & & $\begin{array}{l}0 / 283 \\
(0)\end{array}$ & & \\
\hline \multicolumn{8}{|l|}{$\begin{array}{l}\text { Preterm and } \\
\text { hypertensive } \\
\text { disorders }\end{array}$} \\
\hline Aspirin & $1 / 724(0)$ & $\begin{array}{l}2 \cdot 99 \\
(0 \cdot 12 \\
73 \cdot 32)\end{array}$ & $\begin{array}{l}0 / 445 \\
(0)\end{array}$ & $\begin{array}{l}0.99 \\
(0 \cdot 02 \\
49 \cdot 61)\end{array}$ & $\begin{array}{l}1 / 279 \\
(0)\end{array}$ & $\begin{array}{l}3 \cdot 04 \\
(0 \cdot 12 \\
74 \cdot 38)\end{array}$ & -- \\
\hline Placebo & $0 / 722(0)$ & & $\begin{array}{l}0 / 439 \\
(0)\end{array}$ & & $\begin{array}{l}0 / 283 \\
\text { (0) }\end{array}$ & & \\
\hline \multicolumn{8}{|c|}{ Other Fetal Outcomes of Interest } \\
\hline $\begin{array}{l}\text { Preterm }<34 \\
\text { weeks of } \\
\text { pregnancy }\end{array}$ & & & & & & & \\
\hline
\end{tabular}




\begin{tabular}{|c|c|c|c|c|c|c|c|}
\hline \multirow[t]{2}{*}{ Variable } & \multicolumn{2}{|l|}{ Overall } & \multicolumn{2}{|c|}{ Malaria Negative } & \multicolumn{2}{|c|}{ Malaria Positive } & \multirow{2}{*}{$\begin{array}{l}\text { Aspirin - } \\
\text { Malaria } \\
\text { Interaction } \\
\text { p-value }\end{array}$} \\
\hline & $\mathrm{n} / \mathrm{N}(\%)$ & $\begin{array}{l}\text { PR } \\
(95 \% \\
\text { Cl) }\end{array}$ & $\begin{array}{l}\mathrm{n} / \mathrm{N} \\
(\%)\end{array}$ & $\begin{array}{l}\text { PR } \\
\text { (95\% } \\
\text { Cl) }\end{array}$ & $\begin{array}{l}n / N \\
(\%)\end{array}$ & $\begin{array}{l}\text { PR } \\
(95 \% \\
\text { Cl) }\end{array}$ & \\
\hline Aspirin & $30 / 724(4)$ & $\begin{array}{l}0.88 \\
(0.54 \\
1 \cdot 42)\end{array}$ & $\begin{array}{l}15 / 445 \\
(3)\end{array}$ & $\begin{array}{l}0.74 \\
(0 \cdot 38 \\
1 \cdot 43)\end{array}$ & $\begin{array}{l}15 / 279 \\
(5)\end{array}$ & $\begin{array}{l}1 \cdot 09 \\
(0 \cdot 53 \\
2 \cdot 21)\end{array}$ & 0.40 \\
\hline Placebo & $34 / 722(5)$ & & $\begin{array}{l}20 / 439 \\
(5)\end{array}$ & & $\begin{array}{l}14 / 283 \\
(5)\end{array}$ & & \\
\hline \multicolumn{8}{|c|}{$\begin{array}{l}\text { Birth weight < } \\
2500 \mathrm{~g}\end{array}$} \\
\hline Aspirin & 76/715(11) & $\begin{array}{l}0.94 \\
(0 \cdot 70 \\
1 \cdot 27)\end{array}$ & $\begin{array}{l}37 / 439 \\
(8)\end{array}$ & $\begin{array}{l}0.91 \\
(0.59 \\
1.39)\end{array}$ & $\begin{array}{l}39 / 276 \\
(14)\end{array}$ & $\begin{array}{l}0.98 \\
(0 \cdot 65 \\
1 \cdot 47)\end{array}$ & 0.69 \\
\hline Placebo & $\begin{array}{l}80 / 708 \\
(11)\end{array}$ & & $\begin{array}{l}40 / 432 \\
(9)\end{array}$ & & $\begin{array}{l}40 / 276 \\
(15)\end{array}$ & & \\
\hline \multicolumn{8}{|c|}{$\begin{array}{l}\text { Birth weight < } \\
1500 \mathrm{~g}\end{array}$} \\
\hline Aspirin & $9 / 715(1)$ & $\begin{array}{l}0.69 \\
(0 \cdot 29 \\
1 \cdot 59)\end{array}$ & $\begin{array}{l}1 / 439 \\
(0)\end{array}$ & $\begin{array}{l}0.10 \\
(0.01 \\
0.77)\end{array}$ & $\begin{array}{l}8 / 276 \\
(3)\end{array}$ & $\begin{array}{l}2 \cdot 67 \\
(0 \cdot 71 \\
9 \cdot 95)\end{array}$ & 0.007 \\
\hline Placebo & 13/708 (2) & & $\begin{array}{l}10 / 432 \\
(2)\end{array}$ & & $\begin{array}{l}3 / 276 \\
(1)\end{array}$ & & \\
\hline \multicolumn{8}{|l|}{ Stillbirth } \\
\hline Aspirin & 14/718 (2) & $\begin{array}{l}0.87 \\
(0 \cdot 43 \\
1 \cdot 76)\end{array}$ & $\begin{array}{l}8 / 442 \\
(2)\end{array}$ & $\begin{array}{l}0 \cdot 87 \\
(0 \cdot 34 \\
2 \cdot 23)\end{array}$ & $\begin{array}{l}6 / 276 \\
(2)\end{array}$ & $\begin{array}{l}0 \cdot 87 \\
(0 \cdot 30 \\
2 \cdot 55)\end{array}$ & 0.99 \\
\hline Placebo & 16/712 (2) & & $\begin{array}{l}9 / 432 \\
(2)\end{array}$ & & $\begin{array}{l}7 / 280 \\
(3)\end{array}$ & & \\
\hline \multicolumn{8}{|c|}{$\begin{array}{l}\text { Malaria in late } \\
\text { pregnancy ( } 26- \\
30 \text { weeks) }\end{array}$} \\
\hline Aspirin & $\begin{array}{l}92 / 296 \\
(31)\end{array}$ & $\begin{array}{l}1 \cdot 13 \\
(0 \cdot 88 \\
1 \cdot 45)\end{array}$ & $\begin{array}{l}42 / 177 \\
(24)\end{array}$ & $\begin{array}{l}1 \cdot 42 \\
(0 \cdot 93 \\
2 \cdot 15)\end{array}$ & $\begin{array}{l}50 / 119 \\
(42)\end{array}$ & $\begin{array}{l}0.94 \\
(0 \cdot 70 \\
1 \cdot 26)\end{array}$ & 0.15 \\
\hline Placebo & $\begin{array}{l}83 / 301 \\
(28)\end{array}$ & & $\begin{array}{l}31 / 185 \\
(17)\end{array}$ & & $\begin{array}{l}52 / 116 \\
(45)\end{array}$ & & \\
\hline
\end{tabular}

The association between LDA and preterm birth was similar among the malaria negative (PR $0.89,95 \% \mathrm{Cl}$ $0.59,1.36)$ and the malaria positive group (PR $0.95,95 \% \mathrm{Cl} 0.66,1.38, \mathrm{p}=0.75)$. Perinatal mortality 
occurred in 41 pregnancies in the LDA group and 43 pregnancies in the placebo group (PR $0.95,95 \% \mathrm{Cl}$ $0.63,1.44)$. The association between LDA and perinatal mortality varied between the malaria negative (PR 0.56, 95\% $\mathrm{Cl} 0.31,1.03$ ) and the malaria positive group (PR 1.69, 95\% $\mathrm{Cl} 0.91,3.14$ ), as the model indicated interaction between LDA treatment and malaria infection for perinatal mortality, $(p=0.014)$.

Malaria in late pregnancy was present in 92 pregnancies in the LDA group and 83 pregnancies in the placebo group (PR 1.13, 95\% Cl 0.88, 1.45) (Table 2). The model did not show a significant interaction between LDA treatment and early malaria infection on late malaria status $(p=0.15)$. Outcomes of hypertensive disorders, small for gestational age, vaginal bleeding, antepartum hemorrhage, postpartum hemorrhage, maternal mortality, and the combined outcome of preterm and hypertensive disorders occurred infrequently among the groups. The protective effect of LDA on very low birth weight $(<1500 \mathrm{~g})$ is observed in the malaria negative group but not in the malaria positive group. No evidence of an interaction between LDA and malaria status was observed for any other secondary neonatal outcomes of interest (i.e., interaction terms in model-based analyses were not significant and PRs numerically do not appear to differ).

Preterm birth and perinatal mortality occurred at different frequencies in each research site (Table 3). 
Table 3

Preterm Birth and Perinatal Mortality Outcomes, by Site

\begin{tabular}{|c|c|c|c|c|c|c|c|}
\hline \multirow[t]{2}{*}{ Outcome } & \multicolumn{2}{|l|}{ Overall } & \multicolumn{2}{|c|}{ Malaria Negative } & \multicolumn{2}{|c|}{ Malaria positive } & \multirow{2}{*}{$\begin{array}{l}\text { Aspirin - } \\
\text { Malaria } \\
\text { Interaction } \\
\text { p-value }\end{array}$} \\
\hline & $\begin{array}{l}n / N \\
(\%)\end{array}$ & $\begin{array}{l}\mathrm{PR}(95 \% \\
\mathrm{Cl})\end{array}$ & $\begin{array}{l}\text { N w/o } \\
\text { outcome }\end{array}$ & $\begin{array}{l}\mathrm{PR}(95 \% \\
\mathrm{Cl})\end{array}$ & $\begin{array}{l}\mathrm{N} \text { w/o } \\
\text { outcome }\end{array}$ & $\begin{array}{l}\mathrm{PR}(95 \% \\
\mathrm{Cl})\end{array}$ & \\
\hline \multicolumn{8}{|l|}{ DRC } \\
\hline \multicolumn{8}{|l|}{$\begin{array}{l}\text { Preterm } \\
\text { Delivery }\end{array}$} \\
\hline Aspirin & $\begin{array}{l}44 / 227 \\
(19)\end{array}$ & $\begin{array}{l}0.98 \\
(0 \cdot 68 \\
1 \cdot 41)\end{array}$ & $\begin{array}{l}14 / 87 \\
(16)\end{array}$ & $\begin{array}{l}1.05 \\
(0.53 \\
2 \cdot 10)\end{array}$ & $\begin{array}{l}30 / 140 \\
(21)\end{array}$ & $\begin{array}{l}0.96 \\
(0 \cdot 62, \\
1 \cdot 48)\end{array}$ & 0.84 \\
\hline Placebo & $\begin{array}{l}48 / 242 \\
(20)\end{array}$ & & $\begin{array}{l}13 / 85 \\
(15)\end{array}$ & & $\begin{array}{l}35 / 157 \\
(22)\end{array}$ & & \\
\hline \multicolumn{8}{|l|}{$\begin{array}{l}\text { Perinatal } \\
\text { Mortality }\end{array}$} \\
\hline Aspirin & $\begin{array}{l}18 / 227 \\
(8)\end{array}$ & $\begin{array}{l}1.37 \\
(0.70 \\
2 \cdot 69)\end{array}$ & 3/87 (3) & $\begin{array}{l}0.42 \\
(0.11 \\
1.57)\end{array}$ & $\begin{array}{l}15 / 140 \\
(11)\end{array}$ & $\begin{array}{l}2 \cdot 40 \\
(1.01 \\
5 \cdot 72)\end{array}$ & 0.035 \\
\hline Placebo & $\begin{array}{l}14 / 242 \\
(6)\end{array}$ & & $7 / 85$ (8) & & $\begin{array}{l}7 / 157 \\
(4)\end{array}$ & & \\
\hline \multicolumn{8}{|l|}{ Kenya } \\
\hline \multicolumn{8}{|l|}{$\begin{array}{l}\text { Preterm } \\
\text { Delivery }\end{array}$} \\
\hline Aspirin & $\begin{array}{l}29 / 329 \\
(9)\end{array}$ & $\begin{array}{l}0.95 \\
(0.58 \\
1.55)\end{array}$ & $\begin{array}{l}15 / 199 \\
(8)\end{array}$ & $\begin{array}{l}0.94 \\
(0.48 \\
1 \cdot 84)\end{array}$ & $\begin{array}{l}14 / 130 \\
(11)\end{array}$ & $\begin{array}{l}0.94 \\
(0.46 \\
1.92)\end{array}$ & 0.99 \\
\hline Placebo & $\begin{array}{l}29 / 313 \\
(9)\end{array}$ & & $\begin{array}{l}16 / 199 \\
(8)\end{array}$ & & $\begin{array}{l}13 / 114 \\
(11)\end{array}$ & & \\
\hline \multicolumn{8}{|l|}{$\begin{array}{l}\text { Perinatal } \\
\text { Mortality }\end{array}$} \\
\hline Aspirin & $\begin{array}{l}17 / 329 \\
(5)\end{array}$ & $\begin{array}{l}0.95 \\
(0.49 \\
1 \cdot 83)^{\prime}\end{array}$ & $\begin{array}{l}7 / 199 \\
(4)\end{array}$ & $\begin{array}{l}0.78 \\
(0.30 \\
2.05)\end{array}$ & $\begin{array}{l}10 / 130 \\
(8)\end{array}$ & $\begin{array}{l}1 \cdot 10 \\
(0 \cdot 45 \\
2 \cdot 68)\end{array}$ & 0.62 \\
\hline Placebo & $\begin{array}{l}17 / 313 \\
(5)\end{array}$ & & $\begin{array}{l}9 / 199 \\
(5)\end{array}$ & & $\begin{array}{l}8 / 114 \\
(7)\end{array}$ & & \\
\hline \multicolumn{8}{|l|}{ Zambia } \\
\hline $\begin{array}{l}\text { Preterm } \\
\text { Delivery }\end{array}$ & & & & & & & \\
\hline
\end{tabular}




\begin{tabular}{|c|c|c|c|c|c|c|c|}
\hline \multirow[t]{2}{*}{ Outcome } & \multicolumn{2}{|l|}{ Overall } & \multicolumn{2}{|c|}{ Malaria Negative } & \multicolumn{2}{|c|}{ Malaria positive } & \multirow{2}{*}{$\begin{array}{l}\text { Aspirin - } \\
\text { Malaria } \\
\text { Interaction } \\
\text { p-value }\end{array}$} \\
\hline & $\begin{array}{l}n / N \\
(\%)\end{array}$ & $\begin{array}{l}\mathrm{PR}(95 \% \\
\mathrm{Cl})\end{array}$ & $\begin{array}{l}\mathrm{N} \text { w/o } \\
\text { outcome }\end{array}$ & $\begin{array}{l}\mathrm{PR}(95 \% \\
\mathrm{Cl})\end{array}$ & $\begin{array}{l}\mathrm{N} \text { w/o } \\
\text { outcome }\end{array}$ & $\begin{array}{l}\mathrm{PR}(95 \% \\
\mathrm{Cl})\end{array}$ & \\
\hline Aspirin & $\begin{array}{l}10 / 168 \\
(6)\end{array}$ & $\begin{array}{l}0.76 \\
(0.34 \\
1.70)\end{array}$ & $\begin{array}{l}9 / 159 \\
(6)\end{array}$ & $\begin{array}{l}0.67 \\
(0.30 \\
1.53)\end{array}$ & $1 / 9(11)$ & - & - \\
\hline Placebo & $\begin{array}{l}13 / 167 \\
(8)\end{array}$ & & $\begin{array}{l}13 / 155 \\
(8)\end{array}$ & & $0 / 12(0)$ & & \\
\hline \multicolumn{8}{|l|}{$\begin{array}{l}\text { Perinatal } \\
\text { Mortality }\end{array}$} \\
\hline Aspirin & $\begin{array}{l}6 / 168 \\
(4)\end{array}$ & $\begin{array}{l}0.50 \\
(0 \cdot 19 \\
1 \cdot 29)\end{array}$ & $\begin{array}{l}6 / 159 \\
(4)\end{array}$ & $\begin{array}{l}0.49 \\
(0 \cdot 19 \\
1 \cdot 27)\end{array}$ & $0 / 9(0)$ & - & 1.00 \\
\hline Placebo & $\begin{array}{l}12 / 167 \\
(7)\end{array}$ & & $\begin{array}{l}12 / 155 \\
\text { (8) }\end{array}$ & & $0 / 12(0)$ & & \\
\hline \multicolumn{8}{|c|}{ Notes: $\mathrm{PR}$ is prevalence ratio } \\
\hline PR was nc & Iculatec & i & ich no pa & rinants & rienced th & outcome. & \\
\hline
\end{tabular}

In the DRC, 92/469 (20\%) pregnancies ended in preterm birth, compared to 58/642 (9\%) in Kenya and $23 / 335$ (7\%) in Zambia. Perinatal mortality was observed with an incidence of $7 \%(32 / 469)$ in the DRC, $5 \%$ (34/642) in Kenya, and 5\% (18/335) in Zambia. Effect measure modification was observed in site specific analyses only for perinatal mortality for DRC $(p=0.035)$.

We report hemoglobin levels in late pregnancy based on malaria infection in early pregnancy (Table 4). 
Table 4

Hemoglobin Level, by Malaria Infection and Aspirin exposure

\begin{tabular}{|c|c|c|c|c|c|c|}
\hline \multicolumn{3}{|c|}{ Overall } & \multicolumn{2}{|c|}{ Malaria Negative } & \multicolumn{2}{|c|}{ Malaria positive } \\
\hline $\begin{array}{l}\text { Hemoglobin } \\
\text { cut-point }\end{array}$ & $\begin{array}{l}\mathrm{N}(\mathrm{w} / \text { and } \\
\mathrm{w} / 0 \\
\text { outcome })\end{array}$ & $\begin{array}{l}\mathrm{PR}(95 \% \\
\mathrm{Cl})\end{array}$ & $\begin{array}{l}\mathrm{N} \text { (w/ and } \\
\mathrm{w} / \mathrm{O} \\
\text { outcome) }\end{array}$ & $\begin{array}{l}\mathrm{PR}(95 \% \\
\mathrm{Cl})\end{array}$ & $\begin{array}{l}\mathrm{N}(\mathrm{w} / \text { and } \\
\mathrm{w} / \mathrm{O} \\
\text { outcome) }\end{array}$ & $\begin{array}{l}\mathrm{PR}(95 \% \\
\mathrm{Cl})\end{array}$ \\
\hline \multicolumn{7}{|l|}{$\begin{array}{l}\text { Hemoglobin } \\
\leq 8 \mathrm{~g} / \mathrm{dL}\end{array}$} \\
\hline Aspirin & $11 / 618$ & $\begin{array}{l}0.91 \\
(0.04 \\
2 \cdot 04)\end{array}$ & $3 / 376$ & $\begin{array}{l}0.58 \\
(0 \cdot 14 \\
2 \cdot 43)\end{array}$ & $8 / 242$ & $\begin{array}{l}1 \cdot 16 \\
(0 \cdot 43 \\
3 \cdot 14)\end{array}$ \\
\hline Placebo & $12 / 610$ & & $5 / 364$ & & $7 / 246$ & \\
\hline \multicolumn{7}{|l|}{$\begin{array}{l}\text { Hemoglobin } \\
\leq 9 \mathrm{~g} / \mathrm{dL}\end{array}$} \\
\hline Aspirin & $43 / 586$ & $\begin{array}{l}1 \cdot 06 \\
(0 \cdot 70 \\
1 \cdot 58)\end{array}$ & $10 / 369$ & $\begin{array}{l}0.57 \\
(0 \cdot 27 \\
1 \cdot 23)^{\prime}\end{array}$ & $33 / 217$ & $\begin{array}{l}1 \cdot 34 \\
(0 \cdot 82 \\
2 \cdot 18)\end{array}$ \\
\hline Placebo & $42 / 580$ & & $17 / 352$ & & $25 / 228$ & \\
\hline \multicolumn{7}{|l|}{$\begin{array}{l}\text { Hemoglobin } \\
\leq 10 \mathrm{~g} / \mathrm{dL}\end{array}$} \\
\hline Aspirin & $122 / 507$ & $\begin{array}{l}1 \cdot 01 \\
(0 \cdot 80 \\
1 \cdot 26)\end{array}$ & $49 / 330$ & $\begin{array}{l}1 \cdot 04 \\
(0 \cdot 71 \\
1 \cdot 51)^{\prime}\end{array}$ & 73/177 & $\begin{array}{l}1 \cdot 00 \\
(0 \cdot 76 \\
1 \cdot 31)\end{array}$ \\
\hline Placebo & $120 / 502$ & & $46 / 323$ & & $74 / 179$ & \\
\hline \multicolumn{7}{|l|}{$\begin{array}{l}\text { Hemoglobin } \\
\leq 11 \mathrm{~g} / \mathrm{dL}\end{array}$} \\
\hline Aspirin & $266 / 363$ & $\begin{array}{l}1 \cdot 03 \\
(0 \cdot 90 \\
1 \cdot 17)\end{array}$ & $128 / 251$ & $\begin{array}{l}1 \cdot 07 \\
(0 \cdot 87 \\
1 \cdot 31)\end{array}$ & $138 / 112$ & $\begin{array}{l}1 \cdot 00 \\
(0 \cdot 86 \\
1 \cdot 18)\end{array}$ \\
\hline Placebo & $256 / 366$ & & $117 / 252$ & & $139 / 114$ & \\
\hline
\end{tabular}

Hemoglobin levels in late pregnancy appeared to be similar among women with malaria positive and women that were malaria negative, and LDA treated and untreated groups (Fig. 2).

\section{Discussion}


Our study investigated the potential interaction between LDA and malaria in early pregnancy. We found that malaria in early pregnancy did not modify the effects of LDA on the risk of preterm birth. However, we did observe effect measure modification between LDA and malaria on perinatal mortality. Women without malaria in early pregnancy had lower rates of perinatal mortality when given LDA versus placebo while this protective effect was not observed for women with malaria in early pregnancy. Third trimester hemoglobin levels did not vary based on LDA exposure and malaria status.

Our study adds important knowledge to the lessons learned from the ASPIRIN trial. Within the ASPIRIN trial, we observed a risk ratio of 0.89 (0.81 to 0.98$)$ for preterm birth, among women receiving LDA compared to women receiving placebo, and an absolute reduction of prematurity of $2 \%$.(3) While the ASPIRIN trial was not powered to evaluate a benefit by site, across the 8 sites, the relative risk ranged from $0.66(0.44,1.00)$ to $1.13(0.90,1.42)$, with the highest RR being observed in DRC. It is biologically plausible that the beneficial effect of LDA on preterm birth was modified by malaria given the role of LDA and malaria on placental development.(5) However, our findings did not suggest effect modification on preterm birth by malaria status.

The ASPIRIN trial reported a trend toward significance in regards to a reduction in perinatal mortality (RR $0.86[0.75,1.00])$. We report potential effect measure modification by malaria for the effect of LDA on perinatal mortality, in which women with malaria in pregnancy might not benefit from LDA. This finding was consistent in the DRC in site-specific analyses. It does not appear that effect measure modification through preterm birth explains the perinatal mortality findings. Our convenience sampling strategy resulted in a sample too small to fully explore the effect measure modification. Furthermore, because we restricted our analyses to pregnancies that extended beyond 20 weeks, we might have underestimated the early effects of malaria and LDA on early pregnancy loss (i.e., prior to the gestation of enrollment in the trial).

Treatment or prevention of malaria in pregnancy is a strategy to improve maternal and neonatal outcomes. $(5,13)$ Malaria in pregnancy can exert multiple negative health effects on the mother and the fetus, such as preterm birth, IUGR, and stillbirth.(5) Malaria in pregnancy has also been associated with hypertensive disorders of pregnancy, a condition that might be prevented by LDA treatment in pregnancy. (14) Our cohort had a low incidence of hypertensive disorders of pregnancy (1.4\% in the LDA group and $1.2 \%$ in the placebo group), an incidence that is lower than predicted. Therefore, the joint effects of malaria and LDA on pregnancy outcomes among hypertensive women are still not known.

This study has several strengths. Because of the design of the ASPIRIN trial, we were able to diagnose malaria in early pregnancy, at a median gestational age of 10 weeks. We were also able to study women in three countries with differing levels of malaria endemicity. Despite these strengths, our findings and inferences may have been limited by a relatively small sample size. For example, in our cohort we did not observe an effect of LDA on the outcome of preterm birth. This differed from the overall findings of the ASPIRIN trial, which did show a positive association between LDA and reduction of preterm birth. 
Because of our limited sample, we could have underestimated the contribution of malaria to preterm birth and the beneficial effects of LDA.

Our study emphasizes the importance of evaluating public health interventions within the context in which they will be used, particularly in LMICs where disease burden from infectious diseases is high. Endemic diseases might modify the effect of treatment strategies, by potentiating or eliminating the desired effect. Treatment strategies could be less effective, if not implemented in conjunction with other public health interventions. $(15,16)$ This is particularly true for pre-pregnancy and pregnancy interventions in which packages of interventions are suggested.(17) Since there is an urgent need for timely interventions to improve maternal and newborn health, studies should investigate interventions in diverse settings, where disease endemicity varies, or in conjunction with treatment for concurrent diseases such as malaria.

\section{Conclusion}

Malaria infections during pregnancy are an important cause of preterm birth in many LMICs, especially sub-Saharan African countries where malaria infection in pregnancy is common. Recent trials demonstrate that low dose aspirin (LDA) is an effective strategy to reduce preterm birth, however it is not known if the effect of LDA will be modified based on regional differences in the etiology of preterm birth in low and middle income countries (LMICs). Our study investigated the potential interaction between LDA and malaria in early pregnancy. We found that malaria in early pregnancy did not modify the effects of LDA on the risk of preterm birth. However, malaria modified the benefit of LDA on perinatal mortality, and was associated with less efficacy of LDA to reduce this outcome. Given the urgent need for timely interventions to improve maternal and newborn health, our study highlights the importance of conducting intervention studies in diverse settings, where disease endemicity varies, or in conjunction with treatment for concurrent diseases such as malaria.

\section{Abbreviations}

LMICs - low- and middle-income countries

LDA - low dose aspirin

NICHD - National Institute for Child Health and Human Development

GN - Global Network for Women's and Children's Health Research

DRC - Democratic Republic of Congo

LBW - low birth weight

IUGR - intrauterine growth restriction 
RDTs - rapid diagnostic tests

pfldh - P. falciparum lactate dehydrogenase

qPCR - quantitative polymerase chain reaction

PRs - proportion ratios

ANC - antenatal care

$\mathrm{Cl}$ - confidence interval

\section{Declarations}

\section{Ethics approval and consent to participate}

The methods performed in this study were in accordance with the relevant guidelines and regulations of the Declaration of Helsinki. This study was approved by the relevant ethics committees at the institutions conducting the study at each site prior to the initiation of study activities. The study was also approved by the ethics committees at the partner U.S.-based institutions (University of North Carolina at Chapel Hill, Columbia University, University of Alabama at Birmingham and Indiana University) and by RTI International, the data coordinating center. All women provided informed consent prior to their participation in the sub-study. The ASPIRIN trial was registered in clinicaltrials.gov (NCT02409680).

\section{Consent for publication}

Not applicable.

\section{Availability of data and materials}

De-identified participant data collected for the study are being made available at the NICHD Data and Specimen Hub (https://dash.nichd.nih.gov) with publication. Study protocols, statistical analysis plans and informed consent forms will be made available by the corresponding author (Melissa_bauserman@med.unc.edu) upon request, after approval of a proposal.

\section{Competing interests}

The authors declare that they have no competing interests.

\section{Funding}

This study was funded by grants from the NICHD (UG1 HD076465, UG1HD078437, UG1HD076461). Dr. Marion Koso-Thomas is a project officer within the NICHD Global Network and served a role in oversight of the scientific integrity, monitoring of performance and safety and implementation of protocols. All authors had full access to all the data in the study and accept the responsibility to submit for publication. 


\section{Authors Contributions}

$\mathrm{MB}, \mathrm{EM}, \mathrm{JH}, \mathrm{SM}, \mathrm{CB}$, designed the research (project conception, development of overall research plan and study oversight). MB, AL, AT, JP, EL, FE, WC, EC, RG, SS, SJ, MK, MH, RD, SM conducted the research (hands-on conduct of experiments and data collection). MB, SL, JM, TN analyzed data or performed statistical analyses. $\mathrm{MB}$ and $\mathrm{CB}$ wrote the manuscript. All authors read and reviewed the final version with the exception of SM who passed away during the course of the study.

\section{Acknowledgements}

Not applicable.

\section{References}

1. Chaemsaithong P, Cuenca-Gomez D, Plana MN, Gil MM, Poon LC. Does low-dose aspirin initiated before 11 weeks' gestation reduce the rate of preeclampsia? Am J Obstet Gynecol. 2020;222(5):43750 .

2. Rolnik DL, Wright D, Poon LC, O'Gorman N, Syngelaki A, de Paco Matallana C, et al. Aspirin versus Placebo in Pregnancies at High Risk for Preterm Preeclampsia. N Engl J Med. 2017;377(7):613-22.

3. Hoffman MK, Goudar SS, Kodkany BS, Metgud M, Somannavar M, Okitawutshu J, et al. Low-dose aspirin for the prevention of preterm delivery in nulliparous women with a singleton pregnancy (ASPIRIN): a randomised, double-blind, placebo-controlled trial. Lancet. 2020;395(10220):285-93.

4. Hoffman MK, Goudar SS, Kodkany BS, Goco N, Koso-Thomas M, Miodovnik M, et al. A description of the methods of the aspirin supplementation for pregnancy indicated risk reduction in nulliparas (ASPIRIN) study. BMC Pregnancy Childbirth. 2017;17(1):135.

5. Bauserman M, Conroy AL, North K, Patterson J, Bose C, Meshnick S. An overview of malaria in pregnancy. Semin Perinatol. 2019;43(5):282-90.

6. Steketee RW, Nahlen BL, Parise ME, Menendez C. The burden of malaria in pregnancy in malariaendemic areas. Am J Trop Med Hyg. 2001;64(1-2 Suppl):28-35.

7. Huynh BT, Fievet N, Gbaguidi G, Dechavanne S, Borgella S, Guezo-Mevo B, et al. Influence of the timing of malaria infection during pregnancy on birth weight and on maternal anemia in Benin. Am J Trop Med Hyg. 2011;85(2):214-20.

8. Cottrell G, Mary JY, Barro D, Cot M. The importance of the period of malarial infection during pregnancy on birth weight in tropical Africa. Am J Trop Med Hyg. 2007;76(5):849-54.

9. Briand V, Saal J, Ghafari C, Huynh BT, Fievet N, Schmiegelow C, et al. Fetal Growth Restriction Is Associated With Malaria in Pregnancy: A Prospective Longitudinal Study in Benin. J Infect Dis. 2016;214(3):417-25.

10. Roberge S, Nicolaides KH, Demers S, Villa P, Bujold E. Prevention of perinatal death and adverse perinatal outcome using low-dose aspirin: a meta-analysis. Ultrasound Obstet Gynecol. 2013;41(5):491-9. 
11. Bujold E, Roberge S, Lacasse Y, Bureau M, Audibert F, Marcoux S, et al. Prevention of preeclampsia and intrauterine growth restriction with aspirin started in early pregnancy: a meta-analysis. Obstet Gynecol. 2010;116(2 Pt 1):402-14.

12. Papageorghiou AT, Ohuma EO, Altman DG, Todros T, Cheikh Ismail L, Lambert A, et al. International standards for fetal growth based on serial ultrasound measurements: the Fetal Growth Longitudinal Study of the INTERGROWTH-21st Project. Lancet. 2014;384(9946):869-79.

13. Ota E, da Silva Lopes K, Middleton P, Flenady V, Wariki WM, Rahman MO, et al. Antenatal interventions for preventing stillbirth, fetal loss and perinatal death: an overview of Cochrane systematic reviews. Cochrane Database Syst Rev. 2020;12:CD009599.

14. Mruma HA, McQuillan R, Norrie J. The association of malaria infection and gestational hypertension in Africa: Systematic review and meta-analysis. J Glob Health. 2020;10(2):020417.

15. Althabe F, Belizan JM, McClure EM, Hemingway-Foday J, Berrueta M, Mazzoni A, et al. A populationbased, multifaceted strategy to implement antenatal corticosteroid treatment versus standard care for the reduction of neonatal mortality due to preterm birth in low-income and middle-income countries: the ACT cluster-randomised trial. Lancet. 2015;385(9968):629-39.

16. Sazawal S, Black RE, Ramsan M, Chwaya HM, Stoltzfus RJ, Dutta A, et al. Effects of routine prophylactic supplementation with iron and folic acid on admission to hospital and mortality in preschool children in a high malaria transmission setting: community-based, randomised, placebocontrolled trial. Lancet. 2006;367(9505):133-43.

17. Lassi ZS, Mansoor T, Salam RA, Das JK, Bhutta ZA. Essential pre-pregnancy and pregnancy interventions for improved maternal, newborn and child health. Reprod Health. 2014;11 Suppl 1:S2.

\section{Figures}




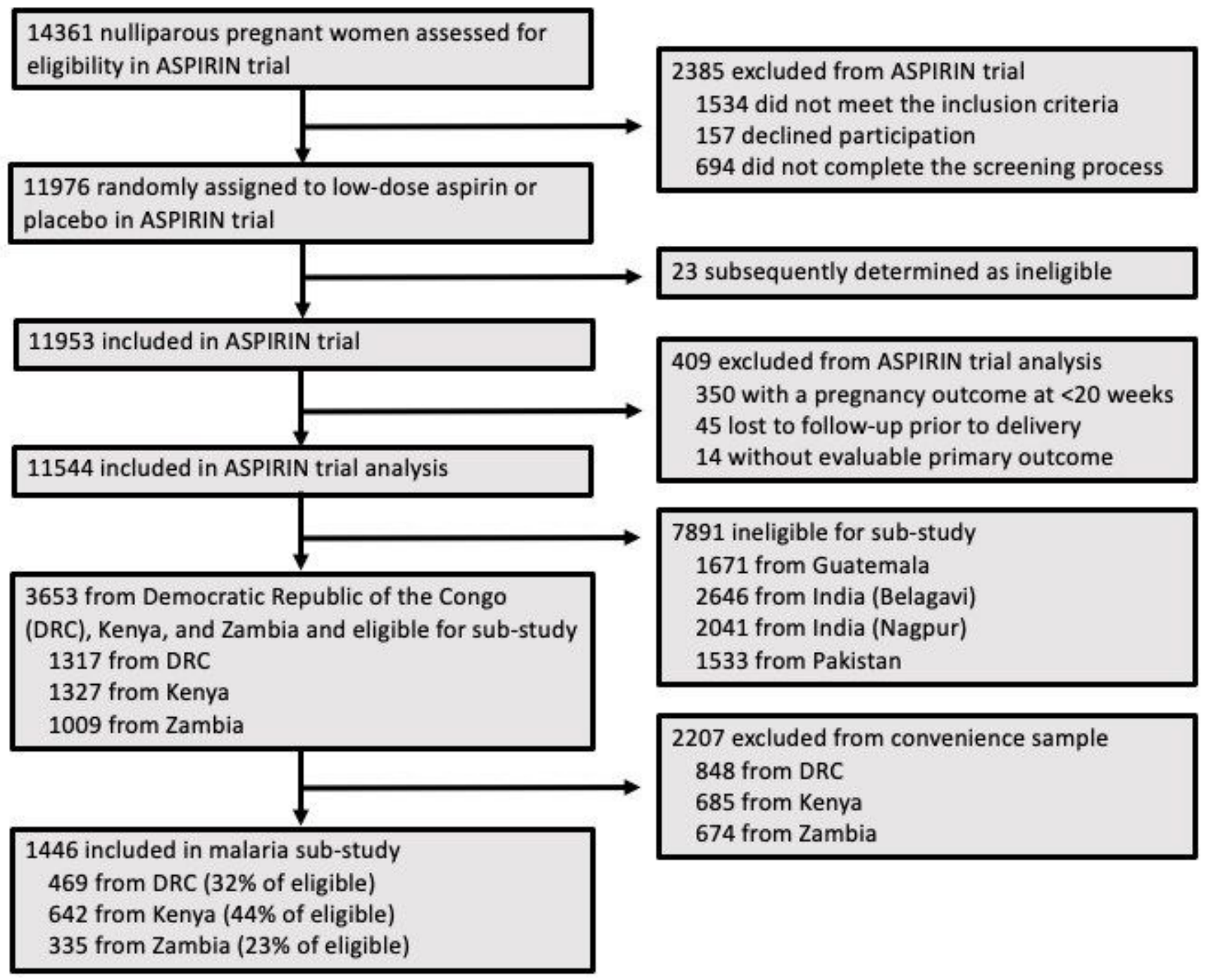

\section{Figure 1}

Study Participants 


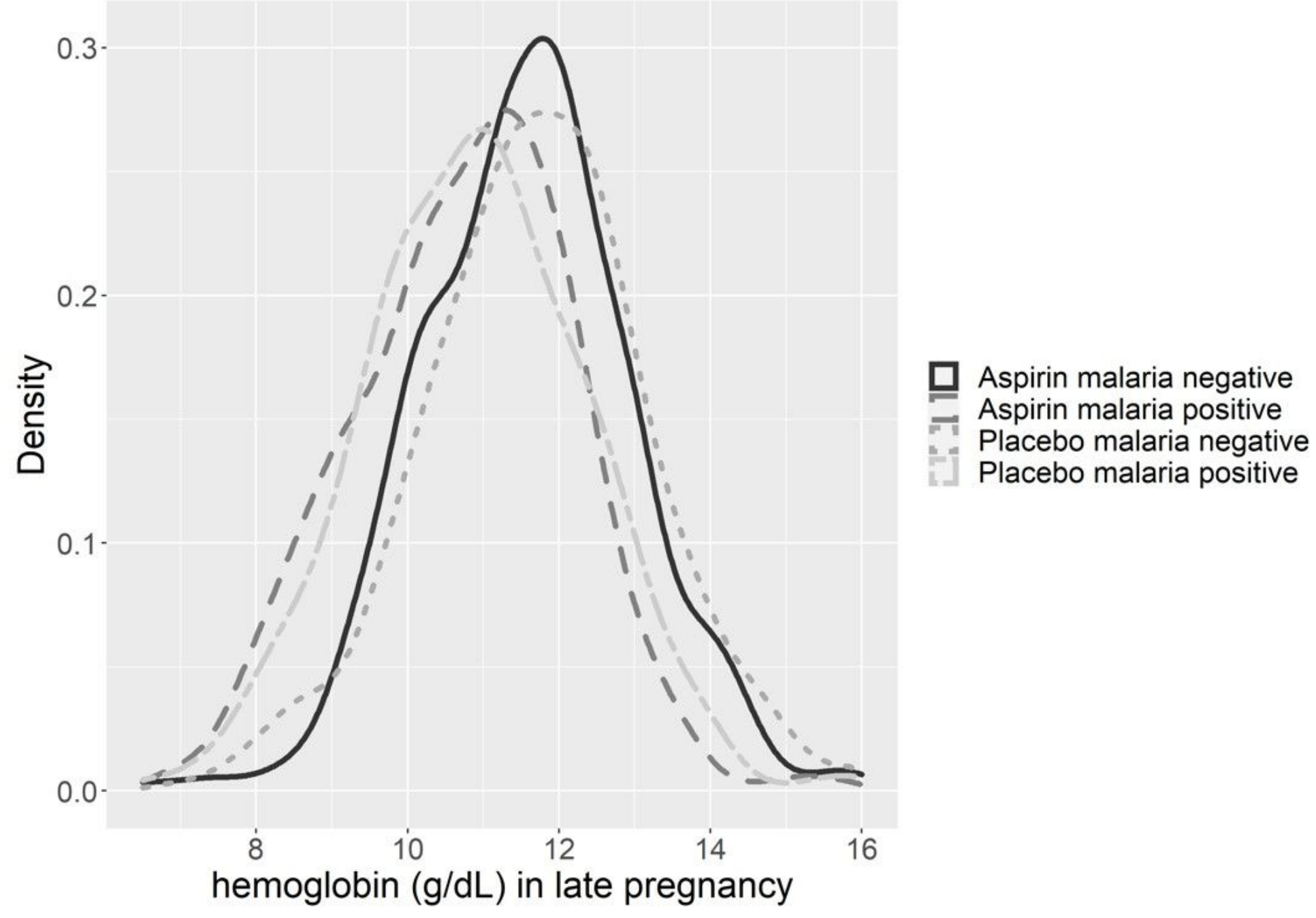

Figure 2

Hemoglobin level in late pregnancy (weeks 26-30), by malaria status in early pregnancy and aspirin exposure 